\title{
Assessing the Effect of Preventive Islanding on Power Grid Resilience
}

DOI:

10.1109/PTC.2019.8810877

\section{Document Version}

Accepted author manuscript

Link to publication record in Manchester Research Explorer

\section{Citation for published version (APA):}

Noebels, M., \& Panteli, M. (2019). Assessing the Effect of Preventive Islanding on Power Grid Resilience. In 2019 IEEE PES PowerTech https://doi.org/10.1109/PTC.2019.8810877

\section{Published in:}

2019 IEEE PES PowerTech

\section{Citing this paper}

Please note that where the full-text provided on Manchester Research Explorer is the Author Accepted Manuscript or Proof version this may differ from the final Published version. If citing, it is advised that you check and use the publisher's definitive version.

\section{General rights}

Copyright and moral rights for the publications made accessible in the Research Explorer are retained by the authors and/or other copyright owners and it is a condition of accessing publications that users recognise and abide by the legal requirements associated with these rights.

\section{Takedown policy}

If you believe that this document breaches copyright please refer to the University of Manchester's Takedown Procedures [http://man.ac.uk/04Y6Bo] or contact uml.scholarlycommunications@manchester.ac.uk providing relevant details, so we can investigate your claim.

\section{OPEN ACCESS}




\section{Assessing the Effect of Preventive Islanding on Power Grid Resilience}

\author{
Matthias Noebels \\ School of Electrical and \\ Electronic Engineering \\ The University of Manchester \\ Manchester, UK \\ Email: matthias.noebels@manchester.ac.uk
}

\author{
Mathaios Panteli \\ School of Electrical and \\ Electronic Engineering \\ The University of Manchester \\ Manchester, UK \\ Email: mathaios.panteli@manchester.ac.uk
}

\begin{abstract}
Cascading faults, caused by extreme weather events or other natural hazards, have been reported to be one of the main mechanism causing widespread blackouts of the power network. A method for preventing the propagation of cascading faults and thus improving power network resilience is intentionally sectionalizing the network into islands. This paper presents a model for simulating cascading faults and evaluates the impact of islanding on island adequacy and network resilience using suitable metrics. The islands are created using two different approaches, a top-down approach based on spectral clustering, and a bottom-up approach. The model is tested and demonstrated in detail on a modified IEEE 30-bus network and the results are quantified using intentional and unintentional load shedding as metrics. The results of the simulations highlight that islanding can effectively prevent cascading faults and thus has a positive effect on network resilience. Results are also provided for the IEEE 57-bus and 118-bus networks to demonstrate the scalability and flexibility of the model.
\end{abstract}

\section{INTRODUCTION}

Cascading faults have been reported to be the main mechanism causing widespread blackouts of the power network [1]. The failure of a transmission line, for example, can lead to overloading of other lines, reactive power problems, or increased system instability. Particularly extreme weather events and natural hazards, such as hurricanes, ice storms, or earthquakes, are the reason for many large-scale power cuts in both developed and developing countries, and the frequency of severe weather events is likely to increase in future [2], [3]. The risk of cascading faults also increases with overall system loading, as the network is operated closer to the maximum ratings and redundancy is decreased.

The ability of the power network to withstand such extraordinary events with a high impact, but a low probability, to "rapidly recover from such disruptive events, and adapt its operation and structure to prevent or mitigate the impact of similar events in the future" [4] is called resilience [5], [6]. One way to increase the network resilience is intentional islanding, which prevents cascading events by splitting the network into separated sections (islands), which are stable and self-adequate [4], [7]. By doing so, vulnerable components are isolated, while the remaining part of the grid is protected and can maintain operation. Any faults happening in one island are no longer able to affect components in other islands, and therefore, cascades are limited to a single island.

In the literature, different types of islanding can be distinguished. Cascading faults by itself often lead to unintentional islands, as the protection system tries to isolate the fault [8]. Because these islands are formed in an uncontrollable way, they are often imbalanced and blackout within short times. Corrective islanding is a way to control the islanding process after a fault or during an ongoing event, in order to stop the propagation of cascading failures and form balanced and adequate islands. In [8], the authors propose an islanding and adaptive load shedding scheme based on predetermined island boundaries for different outage scenarios, such as line and transformer faults. The study shows that these intentional islands perform better witch respect to load shedding compared to unintentional islands. An example for the implementation of corrective islanding is the Manitoba Hydro system in Canada [9], in which the disintegration of the network after a loss of a link is prevented by separating the network into two sections. Preventive islanding, on the other side, refers to intentionally creating islands prior to any faults. Preventive islanding requires a hazard prediction and the identification of vulnerable components, which consequently are isolated in order to reduce the impact of faults. In [4], the positive effect of isolating vulnerable parts of the U.K. transmission network for different storm scenarios on load shedding is shown.

A number of publications deal with finding the best splitting strategy [7], [10]-[12]. Intentional islanding always comes at a cost, as some islands will no longer be able to supply their demand, and consequently, the load in these islands will have to be reduced. The ability of each island to supply its peak demand is called adequacy. While the proposed algorithms optimize various post-fault conditions, including load shedding and restoration capabilities, there is little research on the actual benefit of these methods for network resilience using an adequate metric. The aim of this paper is to investigate the influence of intentional islanding on both the island adequacy and network resilience using suitable metrics. In this study, island adequacy will be quantified using intentional load shedding, i.e. the amount of load that has to be shed in order to maintain adequacy. Quantifying power network resilience is 

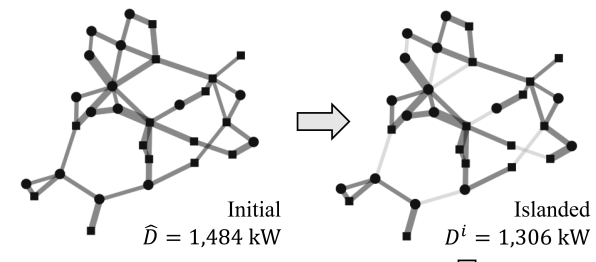

$\sqrt{ }$

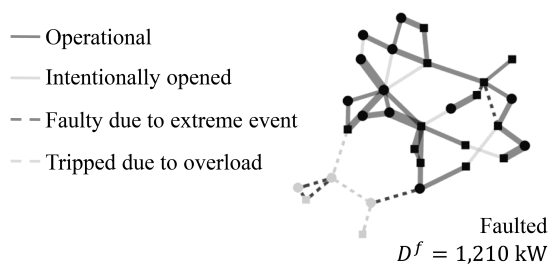

Fig. 1. Intentional islanding of the IEEE 30-bus network into three islands and subsequent simulation of a fault cascade. The supplied load decreases intentionally from initially $\hat{D}$ to $D^{i}$ and unintentionally to $D^{f}$.

the topic of a wide range of publications [13]-[15], however, a common indicator for power network resilience is unintentional load shedding, i.e. the amount of load that is shed due to the loss of lines, generators, or other required network assets [4].

An illustrative example of this concept is given in Figure 1. An initially fully connected and operational network with a total load $\hat{D}$ is intentionally sectionalized into three islands by opening a set of lines (light gray). In order to maintain adequacy, some loads have to be shed and the total load of the network is reduced to $D^{i}$. If now a weather catastrophe damages a number of lines (dashed dark gray), other lines are being overloaded and consequently tripped by the protection system (dashed light gray). After this fault cascade has come to a halt, more loads are shed unintentionally and the network load is further reduced to $D^{f}$.

This rest of this paper is structured as follows: Section II introduces the methodology used for simulating the influence of islanding on adequacy and resilience. The test network and the results for island adequacy and network resilience are presented in Section III. Section IV summarizes the key findings and discusses the further research needed.

\section{Methodology For Assessing IsLAND AdeQuaCy AND NETWORK RESILIENCE}

Figure 2 provides an overview of the simulation procedure, indicating the major steps and iterations, used to assess island adequacy and network resilience. These steps are described in detail in the following sections.

\section{A. Network Description}

A network scenario in this study is defined as the triple

$$
\text { network scenario }=(T, \hat{G}, \hat{D}),
$$

where the topology $\boldsymbol{T}$ is a graph-like pair of $N$ Nodes and $L$ Lines, and $\hat{\boldsymbol{G}}=\left(\hat{G}_{0}, \hat{G}_{1}, \ldots, \hat{G}_{n}\right)$ and $\hat{\boldsymbol{D}}=\left(\hat{D}_{0}, \hat{D}_{1}, \ldots, \hat{D}_{n}\right)$

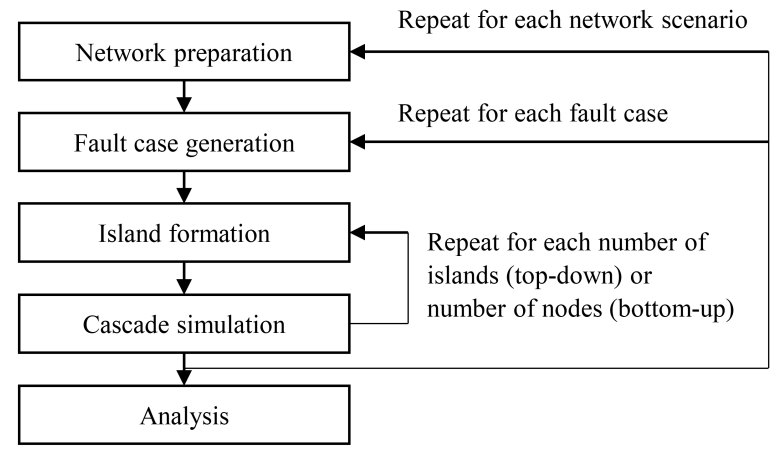

Fig. 2. Flow chart illustrating the simulation steps and iterations of the used methodology for assessing island adequacy and network resilience.

are $N$-dimensional vectors describing the maximum generation and demand, respectively, at each node $n$. The maximum generation $\hat{G}_{n}$ and the maximum demand $\hat{D}_{n}$ at node $n$ are scaled in a way that

$$
\hat{G}=\sum_{n} \hat{G}_{n}=\sum_{n} \hat{D}_{n}=\hat{D}, \text { with } \hat{G}_{n} \geq 0, \hat{D}_{n}>0 \forall n,
$$

i.e. only active power flows are considered and there are no network losses. For each line $L_{n_{1}, n_{2}}$, which connects the corresponding nodes $n_{1}$ and $n_{2}$, a failure probability $p_{n_{1}, n_{2}}^{e}$ for an event $e$ is given. All failure probabilities are normalized to the highest failure probability in the network scenario. The maximum power rating $\hat{P}$ of all lines is set to the same value so that the line with the initially highest power flow is operating at $99 \%$ of its rating:

$$
\hat{P}=\frac{1}{0.99} \cdot \max _{L_{n_{1}, n_{2}}} P_{n_{1}, n_{2}},
$$

where $P_{n_{1}, n_{2}}$ is the calculated power flow in line $L_{n_{1}, n_{2}}$. This simulates that the power system is already operating under extreme conditions when the weather event happens.

The model neglects power system dynamics and transient behavior during switching as well as synchronization between nodes and islands before connecting. The protection system in the islands is assumed to be capable of changing network topology, loops, and reverse power flows.

\section{B. Fault Case Generation}

A fault case is a set of lines which are assumed to be affected by an event $e$, and are therefore not operational any longer. The fault case is later used by the cascade simulation as the initial set of faulty lines. In order to generate a fault case, a weighting factor $w_{n_{1}, n_{2}}^{e} \in(0,1]$ is chosen first, which represents the severity of the $e$ at the location of line $L_{n_{1}, n_{2}}$. The fault case is then determined using

$$
\text { faulty }=\left\{L_{n_{1}, n_{2}} \mid w_{n_{1}, n_{2}}^{e} \cdot p_{n_{1}, n_{2}}^{e}>r_{n_{1}, n_{2}}\right\},
$$

where $r_{n_{1}, n_{2}} \in[0,1)$ is a random number generated for each line individually. 

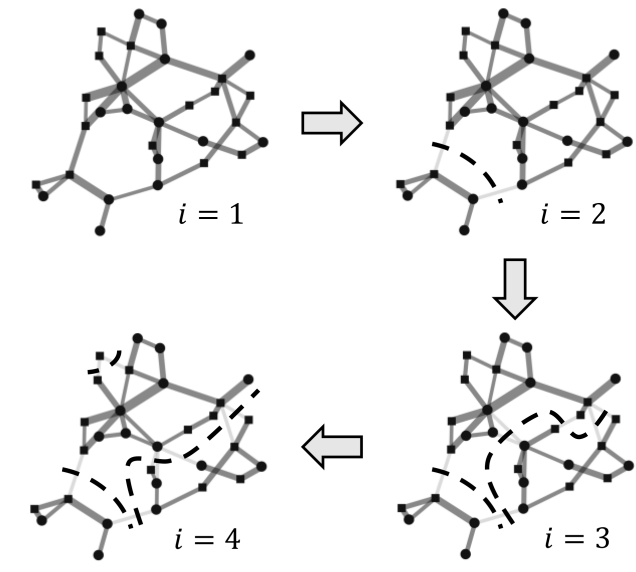

- Closed - Opened - - Island boundaries

Fig. 3. Illustration of the top-down island formation for $1 \leq i \leq 4$ islands.

\section{Island Formation}

Two different methods to investigate islands of different sizes and their impact on network resilience and island adequacy are used in this model, a top-down approach and a bottom-up approach. The different approaches offer insights into different aspects of intentional islanding. While the topdown approach can be used to investigate network resilience depending on number of islands $i$, the bottom-up approach models island adequacy depending on number of nodes $N$ in the island. The top-down approach, which is illustrated in Figure 3, initiates with a connected network, i.e. a large network without any islands. Step by step, the number of islands $i$ is increased until the network only consists of isolated buses. The islanding is done by using spectral clustering, which aims at finding the sparsest cutset to sectionalize a connected graph into a predefined number of islands [16]. The $\mathrm{k}$-medoids algorithm is used to cluster the eigenvectors of the weighted Laplacian matrix. In terms of power networks, the islands are formed by identifying areas in the network which exhibit a minimum power exchange with their environment. This means that the required load shedding due to the islanding process is minimized. By using the top-down approach, the behavior of the total network under different numbers of islands $i$ can be investigated, while the total number of buses $N$ in the network is constant.

The bottom-up approach, on the other hand, simulates the behavior of a single, connected island with an increasing number of nodes $N$, as it is shown in Figure 4. After preparation of the network, one bus is selected randomly as the initial island. Subsequently, all neighbors of the island are identified and one of these neighbors is selected randomly to connect to the island. Hence, the island is growing from a single, isolated bus to a connected network equal to the network it is based on. By using the bottom-up approach, the behaviour of a single island with different numbers of nodes $N$ can be investigated.

After an island has been formed, the optimum power flow

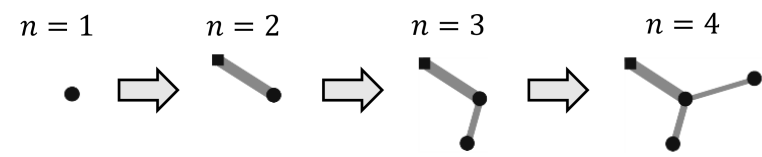

Fig. 4. Illustration of the bottom-up island formation for $1 \leq N \leq 4$ nodes.

(OPF) is calculated, which gives the triple $\left(\boldsymbol{G}^{i}, \boldsymbol{D}^{i}, \boldsymbol{P}^{i}\right)$, where $\boldsymbol{G}^{i}$ and $\boldsymbol{D}^{i}$ are $N$-dimensional vectors describing the dispatched generation $G_{n}^{i} \in\left[0, \hat{G}_{n}\right]$ and demand $D_{n}^{i} \in$ $\left[0, \hat{D}_{n}\right]$ at each node $n$, respectively, after islanding. $\boldsymbol{P}^{i}$ is an $L$-dimensional vector describing the power flow $P_{n_{1}, n_{2}}^{i} \in$ $\left[0, \hat{P}_{n_{1}, n_{2}}\right]$ in each line $L_{n_{1}, n_{2}}$. The intentional load shedding $\Delta D^{i}$ can thus be calculated via

$$
\Delta D^{i}=\sum_{n} D_{n}^{i}-\sum_{n} \hat{D}=D^{i}-\hat{D} .
$$

\section{Cascade Simulation}

The cascade simulation is an iterative algorithm which is inspired by the algorithm used in [4]. The process is illustrated in the flowchart shown in Figure 5. The iteration starts with an initial set of variables $\left(\boldsymbol{G}^{(0)}, \boldsymbol{D}^{(0)}, \boldsymbol{P}^{(0)}\right)=\left(\boldsymbol{G}^{i}, \boldsymbol{D}^{i}, \boldsymbol{P}^{i}\right)$, which describe the dispatched generation and demand as well as power flow immediately after the intentional islanding. Additionally, the lines contained in the fault case are deactivated.

Next, a new OPF is calculated iteratively and for every island $i$ individually, giving, for the $j$ th iteration, the triple $\left(\boldsymbol{G}_{i}^{(j)}, \boldsymbol{D}_{i}^{(j)}, \boldsymbol{P}_{i}^{(j)}\right)$. Because some lines are now not longer operational, this triple will vary from the initial set of variables. For the same reason, also the number of islands $I_{j}$ in the $j$ th iteration may vary and has to be determined for each iteration again. It is assumed that in case of a fault there is not enough time for the network operator to properly redispatch generators and loads in the network based on the changed network topology and the existing line constraints. In order to simulate this behavior, line constraints are ignored and new dispatch constraints for the $j$ th iteration are introduced, which are given by

$$
\begin{aligned}
& G_{n}^{(j)} \in[(1-\delta) \cdot G_{n}^{(j-1)}, \\
&\left.\min \left\{\hat{G}_{n},(1+\delta) \cdot G_{n}^{(j-1)}\right\}\right] \\
& D_{n}^{(j)} \in\left[(1-\delta) \cdot D_{n}^{(j-1)},\right. \\
&\left.\quad \min \left\{\hat{D}_{n},(1+\delta) \cdot D_{n}^{(j-1)}\right\}\right] .
\end{aligned}
$$

This allows the generation and demand only to be changed within a dispatch tolerance range defined by $\delta$. If the imbalance between generation and demand within an island lies outside the dispatch tolerance range, the OPF algorithm does not converge, and the corresponding island is tripped, meaning that all its loads are shed. 


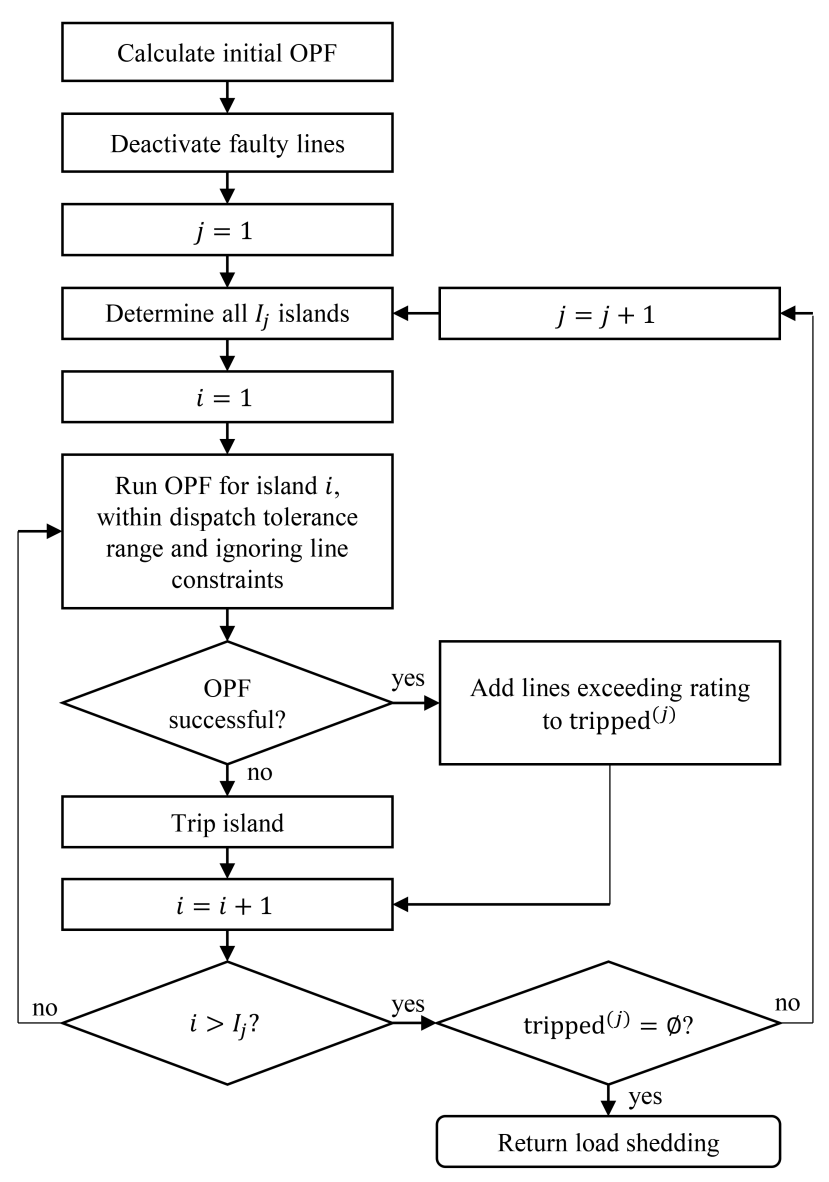

Fig. 5. Flow chart illustrating the fault cascade simulation.

In the next step, the set of lines, which exceed their ratings and are therefore automatically tripped in iteration $j$, is identified via

$$
\operatorname{tripped}^{(j)}=\left\{L_{n_{1}, n_{2}} \mid P_{n_{1}, n_{2}}^{(j)}>\hat{P}\right\} .
$$

If tripped ${ }^{(j)} \neq \emptyset$, a next iteration is executed, until no more lines exceed their rating. In this case, the process is stopped, and the final dispatch is assigned to the triple $\left(\boldsymbol{G}^{f}, \boldsymbol{D}^{f}, \boldsymbol{P}^{f}\right)$. Consequently, the total unintentional load shedding $\Delta D^{u}$ can be calculated via

$$
\Delta D^{u}=\sum_{n} D_{n}^{f}-\sum_{n} D_{n}^{i}=D^{f}-D^{i} .
$$

\section{RESULTS}

\section{A. Test Networks}

The model is developed in Matlab using the MATPOWER toolbox [17]. The following simulations are based on the IEEE 30-bus, 57-bus and 118-bus networks, in order to highlight the flexibility and scalability of the proposed algorithm. In order to increase the diversity of scenarios and to ensure that only situations in which the network is already operating under extreme conditions, only the network topologies $\boldsymbol{T}$ are kept. The values for $\hat{G}$ and $\hat{D}$, i.e. the assignment of generators and demand to the nodes, are generated randomly. To model different levels of availability of distributed generation, only a ratio $p_{g}$ of the available nodes are set to have $\hat{G}_{n} \neq 0$. Finally, $\hat{\boldsymbol{G}}$ and $\hat{\boldsymbol{D}}$ are scaled so that conditions (2) and (3) are fulfilled.

\section{B. Event Modeling}

The event modeled in this simulation is a large storm or hurricane, which is assumed to hit every point of the network with the same strength, i.e. $w_{n_{1}, n_{2}}^{e}=w^{e} \forall n_{1}, n_{2}$. The risk of a line fault is assumed to be proportional to the length of the line. Without loss of generality, it is assumed that all lines have the same impedance per unit length, and the impedance is hence proportional to the length of the line. Hence, the impedance of each line can be used as its failure probability, normalized to the line with the highest impedance in the network.

\section{Island Adequacy}

First, the behavior of island adequacy for different island sizes is analyzed. As mentioned previously, intentional load shedding $\Delta D^{i}$ is used as an indicator for island adequacy. The results for intentional load shedding are given related to the total available load of the network $\hat{D}$ and then averaged over multiple scenarios, e.g. an intentional load shedding of $30 \%$ means that it was only possible to serve $70 \%$ of the available load, while $30 \%$ of the available load had to be switched off.

Figure 6 illustrates the intentional load shedding within a single island with different numbers of nodes $N$ and for two different ratios of generating buses $p_{g}$. The data has been averaged over 200 network scenarios. The islands are formed by the bottom-up approach and are based on the IEEE 30-bus network. It can be seen that the intentional load shedding is the largest when the island consists of only a single node. With growing island size, the intentional load shedding first decreases rapidly until it reaches a critical island size $N_{c}$, after which it decreases slowly until the intentional load shedding reaches zero for a network equal to the initial network. From Figure 6, two key values, the critical island size $N_{c}$ and the residual intentional load shedding $\Delta D_{r}^{i}$, can be determined using linear regression. A first linear regression is calculated approximating the trend in the rapidly changing interval $1 \leq N \leq 3$. The intersection of this line with the node axis gives the critical island size $N_{c}$. A second linear regression is calculated for the interval $N \geq 8$, and the intersection of this line with the load shedding axis gives the residual intentional load shedding $\Delta D_{r}^{i}$. Both regression lines as well as the key values are shown in Figure 6 for the case $p_{g}=0.3$.

The simulation was repeated for multiple steps in the region $0.05 \leq p_{g} \leq 1$ and the values for $N_{c}$ and $\Delta D_{r}^{i}$ have been determined for each of the cases. The results are shown in Figure 7. It can be seen that after an initial decrease, both $N_{c}$ and $\Delta D_{r}^{i}$ stabilize for $p_{g} \geq 0.25$, indicating that for larger ratios of generating buses, the critical island size and the intentional load shedding depend only slightly on $p_{g}$.

\section{Network Resilience}

Next, the impact of island size on network resilience is analyzed. The ratio of generating buses $p_{g}$ has been chosen 


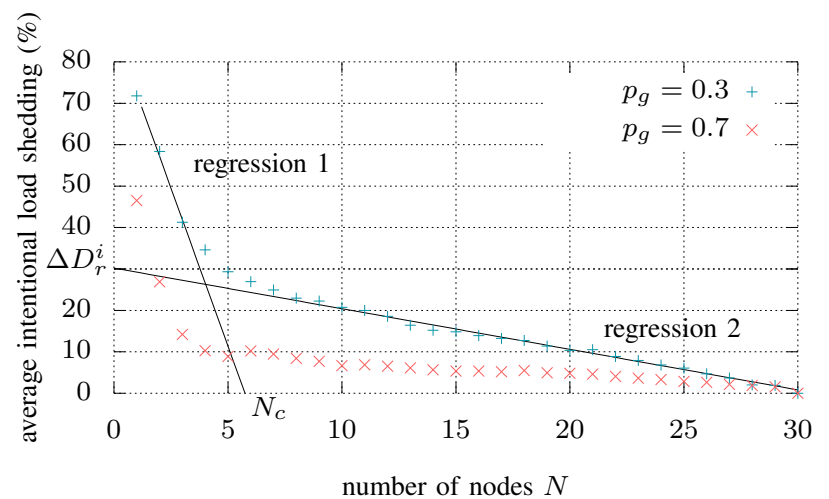

Fig. 6. Average intentional load shedding vs. number of nodes over 200 network scenarios for two different ratios of generating buses $p_{g}$. The figure also shows the two linear regressions and how the key values $N_{c}$ and $\Delta D_{r}^{i}$ are determined.

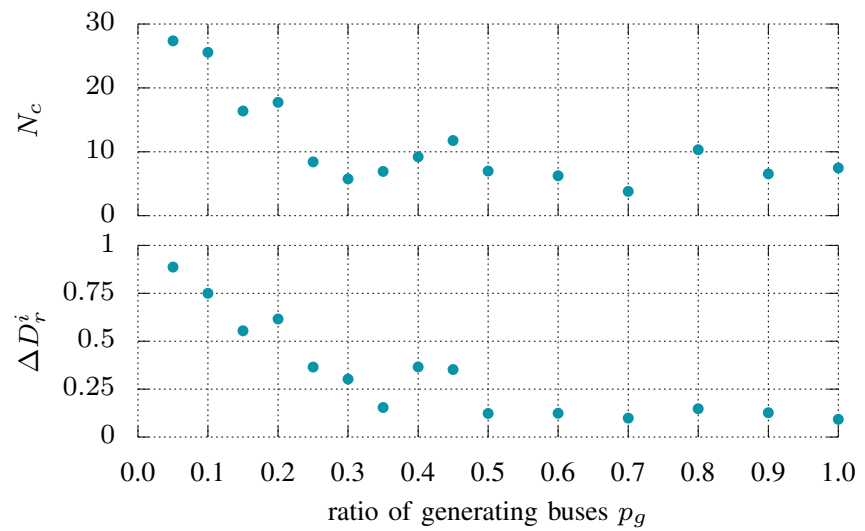

Fig. 7. Critical island size $N_{c}$ and residual intentional load shedding $\Delta D_{r}^{i}$ depending on the ratio of generating buses $p_{g}$.

to be 0.5 in order to reflect a system with a relatively large amount of distributed generation. The total unintentional load shedding $\Delta D^{u}$, which is the difference of load supplied before and after the fault, is used as an indicator for network resilience. The values given in the figures are ratios related to the total network load after islanding $D^{i}$, and are averaged over all network scenarios and fault cases.

Figure 8 shows the average unintentional load shedding in the 30-bus network depending on the number of islands, as generated by the top-down approach, with $\delta=0.1$ and for different weighting factors $w^{e}$, simulating events with different severity. The effect of $\delta$ is analysed in a later step. The data is averaged over 20 network scenarios and 40 fault cases, resulting in 800 individual cases. These numbers have been chosen to reduce computation time by avoiding similar scenarios, because it has been found that the islands formed by spectral clustering depend only to some extent on the specific network scenario, but mainly on the topology.

From the data in Figure 8 it can be seen that the unintentional load shedding decreases for larger number of islands. This is because a higher sectionalized network prevents the spreading of cascading faults, and the amount of affected nodes by the failure of a line is reduced. It can also be seen that

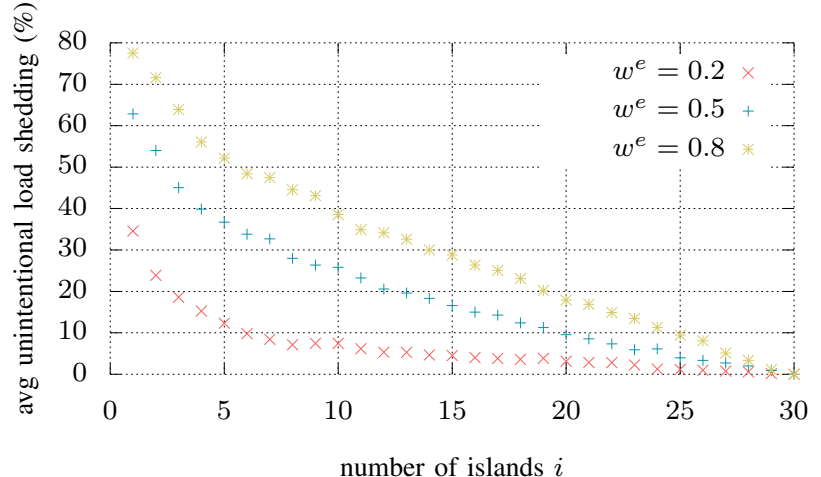

Fig. 8. Average unintentional load shedding vs. number of islands for 20 network scenarios and 40 fault cases for different weighting factors $w^{e}$.

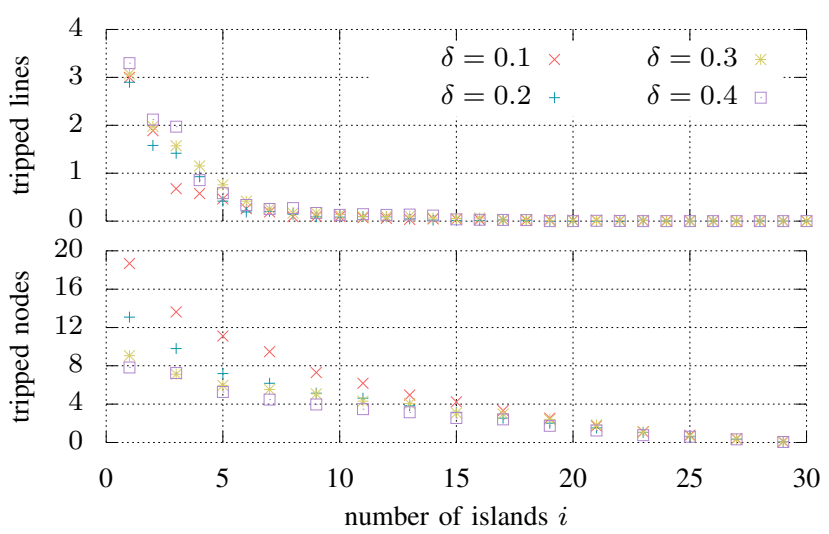

Fig. 9. Dependency of the number of tripped lines and nodes on number of islands $i$ and dispatch tolerance $\delta$.

the unintentional load shedding is larger for higher values for $w^{e}$, as this represents a more severe event with more initially faulty lines.

In the next step, the sensitivity of the network on the dispatch tolerance $\delta$ is examined. For each value of $\delta$ and each number of islands $i$, the average number of lines which were tripped due to overload as well as the average number of nodes which were tripped due to a load imbalance greater than $\delta$ are determined. The event severity is set to be $w^{e}=0.5$. The results are illustrated in Figure 9 and show that the number of both tripped lines and nodes decreases for larger number of islands, proving that islanding efficiently reduces the risk of cascading outages. The number of tripped lines does not depend on $\delta$, because the lines are tripped solely based on the instantaneous power flow and independently of the dispatch tolerance. However, the number of tripped nodes is generally higher for a smaller dispatch tolerance. This is because a lower dispatch tolerance increases the risk of a node with a large imbalance between generation and demand to trip.

Finally, Figure 10 illustrates the behavior of unintentional load shedding for different network topologies, namely the IEEE 30-bus, 57-bus, and 118-bus network. The horizontal axis now display the average number of nodes per island and is drawn in a logarithmic scale. The figure shows a similar trend 


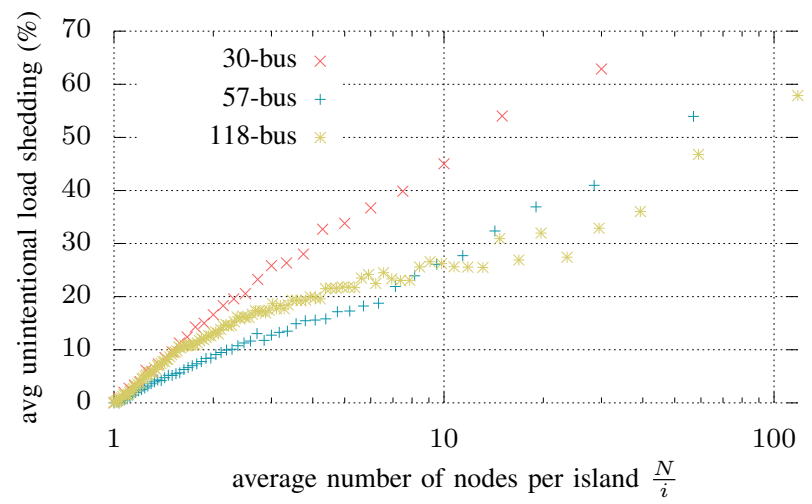

Fig. 10. Average unintentional load shedding vs. average number of nodes per island for the IEEE 30-bus, 57-bus and 118-bus networks.

for all three network topologies. However, the actual amount of unintentional load shedding varies slightly. It is likely that these variances are due to different degrees of interconnectivity of the networks. These topological differences are not part of this study.

\section{CONCLUSION}

This paper presented intentional islanding as a method for preventing the spreading of cascading faults in a power network and thus reducing the impact of catastrophic events. A model for simulating cascading faults was introduced and tested on three different network sizes and topologies with the aim of evaluating the effect of preventive islanding on island adequacy and network resilience. The simulations have shown that increasing the number of islands in a network increases the network resilience. However, islands with a smaller size have a higher imbalance of supply and demand, and therefore require more intentional load shedding. Consequently, it has been proven that intentional islanding prevents cascading outages and that it can be beneficial for increasing the resilience of a power network in case of a catastrophic event.

Islanding requires sufficient amount of distributed generation to maintain adequacy. In case of unpredictable, e.g. renewable, energy sources, adequacy might be violated after islanding. This issue could be overcome by utilizing energy storage systems or implementing other smart control strategies for dealing with the variability of intermittent renewable energy sources.

The decision of a network operator to sectionalize a network must be based on two aspects: On the one hand, sectionalizing comes at an unavoidable cost, because some loads have to be shed in order to maintain adequacy. This can be achieved by using existing smart grid capabilities, such as demand-side management. The network operator can inform its customers prior to the event and incentivize them to reduce their demand accordingly. On the other hand, if the network operator has sectionalized the network, there is a reduced risk of unintentional load shedding in case of a fault. Although the actual amount of reduced unintentional load shedding is not predictable and depends on the actual event, its reduction is always beneficial for the network operator.
The benefit and attractiveness of intentional islanding can be increased in several ways. Future work in this area includes better predictions of upcoming extreme weather events and the implementation of intelligent islanding algorithms that can balance demand and generation in a better way.

\section{ACKNOWLEDGMENT}

The author acknowledges financial support from EPSRC (EP/L016141/1) through the Power Networks Centre for Doctoral Training.

\section{REFERENCES}

[1] D. P. Nedic, I. Dobson, D. S. Kirschen, B. A. Carreras, and V. E. Lynch, "Criticality in a cascading failure blackout model," International Journal of Electrical Power and Energy Systems, vol. 28, no. 9 SPEC. ISS., pp. 627-633, 2006.

[2] P. Hines, J. Apt, and S. Talukdar, "Trends in the history of large blackouts in the United States," IEEE Power and Energy Society 2008 General Meeting: Conversion and Delivery of Electrical Energy in the 21st Century, PES, vol. 15213, pp. 1-8, 2008.

[3] A. Kenward and U. Raja, "Blackout: Extreme Weather, Climate Change and Power Outages," Climate Central, Tech. Rep., 2014.

[4] M. Panteli, D. N. Trakas, P. Mancarella, and N. D. Hatziargyriou, "Boosting the Power Grid Resilience to Extreme Weather Events Using Defensive Islanding," IEEE Transactions on Smart Grid, vol. 7, no. 6, pp. 2913-2922, 2016.

[5] A. R. Berkeley III and M. Wallace, "A framework for establishing critical infrastructure resilience goals: Final goals and recommendations," Nat. Infrastruct. Advisory Council (NIAC), Washington, DC, USA, Tech. Rep., 2010

[6] M. Chaudry, P. Ekins, K. Ramachandran, A. Shakoor, J. Skea, G. Strbac, X. Wang, and J. Whitaker, "Building a Resilient UK Energy System," London, UK, 2011.

[7] Y. Wu, Y. Tang, B. Han, and M. Ni, "A topology analysis and genetic algorithm combined approach for power network intentional islanding," International Journal of Electrical Power and Energy Systems, vol. 71, pp. 174-183, 2015.

[8] H. Dola and B. Chowdhury, "Intentional islanding and adaptive load shedding to avoid cascading outages," 2006 IEEE Power Engineering Society General Meeting, 2006.

[9] B. A. Archer and J. B. Davies, "System Islanding Considerations for Improving Power System Restoration at Manitoba Hydro," in Canadian Conference on Electrical and Computer Engineering (IEEE CCECE 2002), 2002, pp. 60-65.

[10] K. Sun, D. Z. Zheng, and Q. Lu, "Splitting strategies for islanding operation of large-scale power systems using OBDD-based methods," IEEE Transactions on Power Systems, vol. 18, no. 2, pp. 912-923, 2003.

[11] J. Q. Tortos and V. Terzija, "Controlled islanding strategy considering power system restoration constraints," IEEE Power and Energy Society General Meeting, pp. 1-8, 2012.

[12] A. Peiravi and R. Ildarabadi, "A fast algorithm for intentional islanding of power systems using the multilevel kernel k-means approach," Journal of Applied Sciences, vol. 9, no. 12, pp. 2247-2255, 2009.

[13] D. A. Reed, K. C. Kapur, and R. D. Christie, "Methodology for assessing the resilience of networked infrastructure," IEEE Systems Journal, vol. 3 , no. 2, pp. 174-180, 2009.

[14] S. Chanda, A. K. Srivastava, M. U. Mohanpurkar, and R. Hovsapian, "Quantifying power distribution system resiliency using code based metric," 2016 IEEE International Conference on Power Electronics, Drives and Energy Systems (PEDES), pp. 1-6, 2016.

[15] M. Panteli, P. Mancarella, D. N. Trakas, E. Kyriakides, and N. D. Hatziargyriou, "Metrics and Quantification of Operational and Infrastructure Resilience in Power Systems," IEEE Transactions on Power Systems, vol. 32, no. 6, pp. 4732-4742, 2017.

[16] L. Ding, F. M. Gonzalez-longatt, P. Wall, and V. Terzija, "Two-Step Spectral Clustering Controlled Islanding Algorithm,' IEEE Transactions on Power Systems, vol. 28, no. 1, pp. 75-84, 2013.

[17] R. D. Zimmerman, C. E. Murillo-Sanchez, and R. J. Thomas, "MATPOWER: Steady-State Operations, Planning, and Analysis Tools for Power Systems Research and Education," IEEE Transactions on Power Systems, vol. 26, no. 1, pp. 12-19, 2011. 